\title{
IMPACTO EN ASTURIAS DE LAS URGENCIAS DE ATENCIÓN PRIMARIA SOBRE LAS HOSPITALARIAS. UN ANÁLISIS DE COINTEGRACIÓN DE SERIES TEMPORALES (*)
}

\author{
David Oterino de la Fuente (1,2), JF Baños Pino (3), V Fernández Blanco (3), A Rodríguez-Álvarez \\ (1) Departamento de Medicina Preventiva y Salud Pública, Universidad de Oviedo. \\ (2)Fundación Instituto de Investigación en Servicios de Salud. \\ (3) Departamento de Economía, Universidad de Oviedo. \\ (*) Este trabajo forma parte de un proyecto de investigación que obtuvo concurrencialmente la Beca de la Asociación de \\ Economía de la Salud de Investigación en Economía de la Salud 2001, otorgada por esta asociación en su Xa edición.
}

\section{RESUMEN}

Fundamento: La mayor accesibilidad a los puntos de atención continuada (PAC) de la atención primaria podría disminuir las visitas en los Servicios de Urgencias Hospitalarias (SUH). En este estudio se analiza si existe sustituibilidad entre las urgencias de Atención Primaria y Hospitalaria.

Métodos: Se analiza la totalidad de las visitas urgentes ( $n=6.454 .034)$ realizadas en los SUH de los hospitales y PAC de Atención Primaria de Asturias y de cada una de las áreas sanitarias entre 1994 y 2001. Se construyeron las series temporales con frecuencias mensuales para Asturias y cada una de las áreas y se realizó un análisis de cointegración para evaluar si existe sustituibilidad entre ambas series.

Resultados: Se observó un incremento medio anual de las urgencias totales en Asturias del 6,2\% (PAC: 7,8\%; SUH: $5,1 \%$ ), con diferente crecimiento entre las áreas sanitarias. En el análisis de cointegración de las series temporales no se detectó sustituibilidad entre las urgencias de atención primaria y hospitalaria para Asturias y para las áreas sanitarias, salvo en el área sanitaria de Oviedo, donde una tasa de crecimiento del $10 \%$ en primaria reduciría un $2,7 \%$ las urgencias hospitalarias.

Conclusiones: La mayor accesibilidad a los PAC de Atención Primaria incrementa su utilización sin reducir las visitas en los SUH. En consecuencia, el incremento de recursos en Atención Primaria no parece constituir una alternativa eficaz para disminuir las visitas en los SUH.

Palabras Clave: Servicios de urgencias atención primaria. Servicios de urgencias hospitalarias. Análisis de cointegración. Series temporales.

\section{Correspondencia}

David Oterino de la Fuente

Fundación Instituto de Investigación en Servicios de Salud.

El Puerto 46. 33457 Sta. Ma del Mar. Asturias

Correo electrónico: davidoterino@wanadoo.es

\section{ABSTRACT \\ Impact in Asturias of Primary Care Emergencies on Hospital Emergencies for the 1994-2001 Period. A Time Series Cointegration Analysis}

Background: Greater accessibility to the primary care continuing care points (CCP's) could reduce the visits to the Hospital Emergency Services (HES's). This study analyses whether Primary Care can replace and Hospital Services in emergencies.

Methods: All of the emergency visits $(n=6.454 .034)$ made to the HES's and Primary Care CCP's in Asturias and of each one of the healthcare districts within the 1994-2001 period were calculated. The time series were constructed with monthly frequencies for Asturias and each one of the districts, a cointegration analysis having been made to assess whether the two series are inter-replaceable.

Results: A mean annual increase of the total number of emergencies in Asturias of $6.2 \%$ (CCP: 7,8\%; HES: $5.1 \%$ ) was found, with different growth among the healthcare districts. In the time series cointegration analysis, no replaceability was found between the primary care and hospital emergencies for Asturias and for the healthcare districts, except for the healthcare district of Oviedo, where a $10 \%$ growth rate in primary would lower hospital emergencies by $2.7 \%$.

Conclusions: The greater accessibility to the Primary Care CCP's increases the use thereof without reducing the visits to the HES's. Therefore, the increase in Primary Care resources does not seem to be an effective alternative for reducing the visits to the HES's.

Keywords: Emergency visits. Primary care. Accident and emergency departments. co-integration Time-series analysis. 


\section{INTRODUCCIÓN}

El crecimiento de las visitas urgentes hospitalarias ha dado lugar a múltiples estudios sobre los factores relacionados con la asistencia y la inadecuación de las visitas y se han analizado numerosas intervenciones puestas en práctica para disminuir las visitas no urgentes (inadecuadas, no justificadas) en los Servicios de Urgencias Hospitalarios (SUH) y tratar a los pacientes en el lugar, en el tiempo y con los medios más adecuados ${ }^{1-6}$.

Una de las intervenciones que intuitivamente parece razonable para disminuir las visitas no urgentes en los SUH es mejorar la accesibilidad a la Atención Primaria (AP) -implantación de la AP para grupos de población o zonas geográficas donde no existía, continuidad de la asistencia, implantación de programas preventivos $^{7-11}$ - y/o la accesibilidad a los servicios de urgencia de atención primaria -incremento el número de Puntos de Atención Continuada (PAC)-, walk-in centres, los out-of-hours health services, consulta telefónica- y los recursos existentes -ampliación de horario, aumento de personal sanitario y de recursos técnicos y de transporte ${ }^{12-17}$. No obstante, la revisión de la efectividad de las intervenciones/innovaciones que mejoran la atención primaria no muestran evidencias concluyentes: en las dirigidas a mejorar la accesibilidad de la atención primaria en general parece observarse una efectividad mínima, mientras que en las dirigidas a la accesibilidad a los servicios de urgencias de AP muestran escasa o nula efectividad. En todo caso hay que tener en cuenta que están realizadas en contextos sanitarios donde la atención primaria tiene diferentes grados de desarrollo o en periodos excesivamente cortos que no se han seguido en el tiempo, con diferentes metodologías y grupos de población de distintas culturas.
Recientemente ha sido publicado un artículo de los mismos autores ${ }^{12}$ que evalúa, mediante el análisis de cointegración de las series temporales, las visitas urgentes a los SUH y PAC circunscrito a un área sanitaria (AS) de Asturias de 80.000 habitantes entre 1992 y 1999 , para evaluar si existe capacidad de sustitución entre las mismas. En esta línea, el presente trabajo analiza las series mensuales de las visitas urgentes a los PAC y SUH en la Comunidad Autónoma de Asturias, durante el periodo 1994-2001, con la misma metodología (análisis de cointegración de las series temporales) y el objetivo de evaluar si el incremento de las urgencias en los PAC disminuye las visitas de los SUH, es decir, si la mejora de la accesibilidad a la AP se asocia a cambios en la tendencia en la utilización de los SUH.

\section{SUJETOS Y MÉTODOS}

Diseño. Estudio de las series temporales de urgencias en atención primaria y hospitalaria del Principado de Asturias desde 1994 al 2001.

Localización y recursos. El Principado de Asturias contaba en 2001 con una población de 1.062.998 habitantes. Demográficamente la última década se ha caracterizado por un crecimiento vegetativo negativo y un fuerte proceso de envejecimiento, con un $21,7 \%$ de personas mayores de 65 años en el 2001. Su sistema sanitario está estructurado en 8 Áreas Sanitarias, cada una con un hospital público de referencia donde se ubica el Servicio de Urgencias Hospitalario (SUH). Igualmente cada Zona Básica de Salud (ZBS) dispone de un Punto de Atención Continuada (PAC) cuyo horario de funcionamiento es de 17 a 22 horas en los días laborables, y de 8 a 22 horas los festivos y fines de semana, aunque en los PAC rurales la asistencia incluye el hora- 
rio nocturno (de 22 a 8 horas). En atención primaria el personal médico y de enfermería que atiende las urgencias es el del Equipo de Atención Primaria del centro o es personal de refuerzo contratado expresamente para atender urgencias. Algunas ZBS disponen también de un Servicio Ordinario de Urgencias (SOU) dedicado a la asistencia de urgencias conforme al modelo sanitario previo a la reforma de 1984, con un horario de 17 a 8 horas.

Población. Todas las visitas urgentes atendidas en los servicios de urgencia de los hospitales públicos y en los PAC de atención primaria de Asturias en el periodo 1994-2001.

Fuentes de datos. La información correspondiente a los hospitales procede de la monografía Asistencia especializada. Actividad 2001. Evolucion de indicadores 1991-2001'18 y de los Registros de Urgencias de los hospitales Jove y Grande Covián. Por lo que respecta a este último centro, inaugurado en 1997 para atender poblaciones que previamente estaban asignadas al área de Oviedo, sólo hay información disponible desde el año 1999. Los datos de atención Primaria fueron facilitados directamente por las Gerencias de sus Áreas Sanitarias.

Análisis: Se calcularon las tasas de urgencias brutas por 1000 habitantes para cada mes, año, área sanitaria y total de Asturias (Tasa de urgencias = número de urgencias/población), utilizando como denominador la población de cada área sanitaria según el padrón municipal de cada año. Para cada área y nivel asistencial se describe la evolución anual del número de urgencias y sus porcentajes de crecimiento en el periodo 19942001 (1999-2001 para el Área de Arriondas). Con las visitas urgentes mensuales se construyeron las series temporales de A. P. y hospitalaria correspondientes a cada área sanitaria y Asturias.

Análisis de cointegración de series temporales: El análisis de cointegración es un instrumento econométrico relativamente reciente que persigue identificar relaciones estables, o de largo plazo, entre dos o más variables utilizando series temporales, esta metodología está amplia y detalladamente descrita en un artículo previo $^{12}$.

En primer lugar, al haberse empleado series mensuales se realiza un análisis para contrastar para cada una de ellas la existencia de raíces unitarias, en las distintas frecuencias estacionales. Todas las variables han sido transformadas en términos logarítmicos, tomándose luego una diferencia regular y otra estacional, aplicándose además procesos $\mathrm{AR}(3)$ x AR(1)12 a fin de conseguir que los residuos sean ruido blanco ${ }^{19}$.

Para eliminar las raíces estacionales se aplicó el método descrito por Hylleberg ${ }^{20}$, posteriormente se aplicaron los tests de cointegración estándar, en concreto, el que está basado en el contraste descrito por Engle y Granger ${ }^{21}$, que aplica el test de Dickey-Fuller aumentado, complementado con el procedimiento de máxima verosimilitud de Johansen ${ }^{22}$. Para determinar el número de vectores de cointegración (o posibles relaciones de largo plazo) se ha utilizado el test de la traza ajustado para muestras pequeñas, como sugiere Reimers $^{23}$.

En el área sanitaria de Oviedo se tiene en cuenta el primer resultado al apreciarse una relación de cointegración, aunque su signo es distinto dependiendo del contraste aplicado, como las propiedades del procedimiento de Johansen (tabla 3) son superiores a las de Engle (tabla 4) se tiene encuenta el primer resultado. Para 
corroborarlo se ha estimado una relación a largo plazo directamente por métodos no lineales, como sugiere Banergee ${ }^{24}$.

\section{RESULTADOS}

Entre 1994 y 2001 se realizaron casi 6,5 millones de visitas urgentes en Asturias $(n=6.454 .034)$, (PAC: 56,2\%; SUH: $43,8 \%)$. Las urgencias totales crecieron un $43,4 \%$ (incremento medio anual del
6,2\%; PAC: 7,8\%; SUH: 5,1\%). La atención urgente creció en todas las áreas sanitarias en general, aunque no en todas lo hizo más en AP que en los hospitales, no siendo este crecimiento homogéneo entre áreas, tanto en AP (con incrementos que van desde el $31 \%$ en Mieres a más del $80 \%$ en Oviedo o Cangas, dejando a un lado el área de Arriondas, afectada por la apertura de un SUH), como en hospitales (que van desde el 7,8\% en Oviedo al 68\% en Avilés). En la tabla 1 se muestran las

Tabla 1

Tasas crudas de visitas urgentes por 1000 habitantes en cada área sanitaria (evolución 1994-2001)

\begin{tabular}{|c|c|c|c|c|c|c|c|c|c|c|}
\hline \multirow{3}{*}{ Área } & \multicolumn{5}{|c|}{ Urgencias Atención Primaria } & \multicolumn{5}{|c|}{ Urgencias Hospitalarias } \\
\hline & \multicolumn{2}{|c|}{1994} & \multicolumn{2}{|c|}{2001} & \multirow{2}{*}{$\begin{array}{c}\% \text { 94-01 } \\
\text { Tasa }\end{array}$} & \multicolumn{2}{|c|}{1994} & \multicolumn{2}{|c|}{2001} & \multirow{2}{*}{$\begin{array}{c}\% \text { 94-01 } \\
\text { Tasa }\end{array}$} \\
\hline & & $\mathbf{n}$ & Tasa & $\mathbf{n}$ & & & $\mathbf{n}$ & Tasa & $\mathbf{n}$ & \\
\hline 1 Jarrio & 24.421 & 406,8 & 31.188 & 568,0 & 39,63 & 14.582 & 242,9 & 20.862 & 379,9 & 56,40 \\
\hline 2 Cangas & 13.719 & 338,7 & 22.351 & 625,2 & 84,59 & 9.548 & 235,7 & 12.577 & 351,8 & 49,26 \\
\hline 3 Avilés & 45.265 & 269,4 & 69.695 & 438,9 & 62,92 & 39.923 & 237,6 & 63.418 & 399,4 & 68,10 \\
\hline 4 Oviedo & 63.054 & 199,9 & 114.895 & 366,4 & 83,29 & 111.191 & 352,5 & 119.182 & 380,1 & 7,83 \\
\hline 5 Gijón & 80.476 & 272,2 & 130.180 & 442,7 & 62,64 & 82.682 & 279,6 & 111.933 & 380,7 & 36,16 \\
\hline 6 Arriondas & 28.827 & 489,5 & 31.113 & 575,2 & 17,51 & 0 & 0 & 16.422 & 303,6 & $* 27,40$ \\
\hline 7 Mieres & 49.696 & 425,2 & 65.524 & 558,9 & 31,44 & 22.379 & 271,9 & 25.026 & 347,0 & 27,62 \\
\hline 8 Nalón & 36.234 & 529,1 & 43.496 & 758,8 & 43,42 & 23.170 & 238,3 & 27.002 & 289,8 & 21,64 \\
\hline Asturias & 341.692 & 305,7 & 508.442 & 568,0 & 54,70 & 303.475 & 271,5 & 396.422 & 368,7 & 35,78 \\
\hline
\end{tabular}

* En el caso del Hospital de Arriondas, inaugurado en 1997, la base para el incremento de tasas se refiere al primer año con datos disponibles (1999: 13059 visitas; 238,3 por 1000 habitantes).

Figura 1

Evolución en Asturias de la tasa bruta de urgencias por 100000 habitantes, 1994-2001

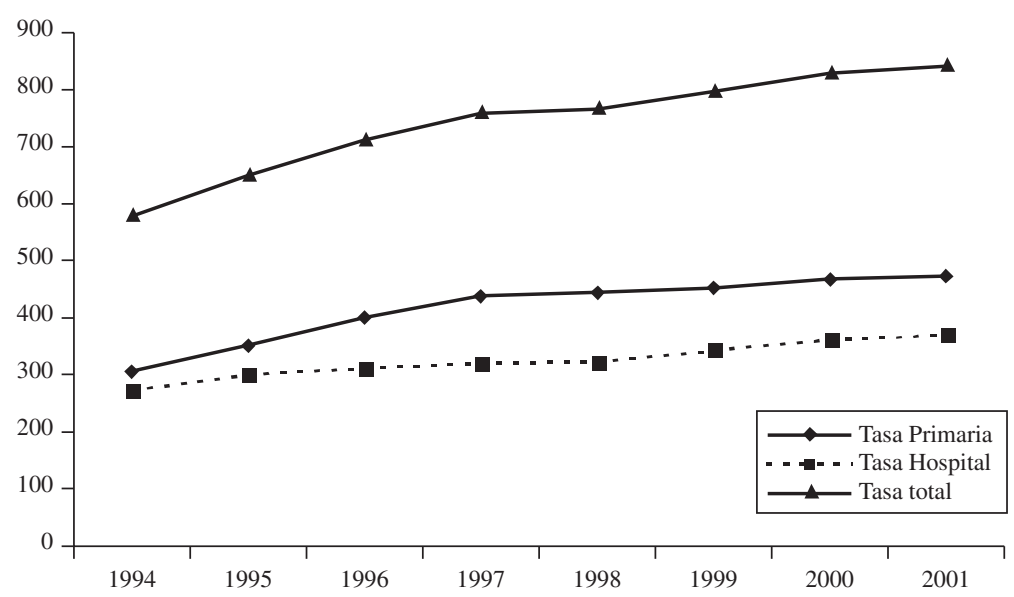


Tabla 2

Contraste de raíces unitarias Series mensuales visitas urgentes en logaritmos Hipótesis nula: SI(2,1)

\begin{tabular}{|c|c|c|c|c|c|c|c|c|c|}
\hline \multicolumn{10}{|c|}{ PUNTOS DE ATENCIÓN CONTINUADA (PAC) } \\
\hline Área Sanitaria & Jarrio & Cangas & Avilés & Oviedo & Gijón & Arriondas & Nalón & Mieres & Asturias \\
\hline Regresión: & $\mathbf{n}$ & $c, t, d$ & n & nn & $\mathbf{c , d}$ & n & n & $\mathbf{c , d}$ & \\
\hline \multicolumn{10}{|l|}{ Estadíst-t: } \\
\hline$\sigma_{1}$ & 1,03 & 1,65 & 1,29 & 0,78 & 1,35 & 1,73 & 0,60 & 1,60 & 1,35 \\
\hline$\sigma_{2}$ & $-0,47$ & $-1,79$ & $-2,02 *$ & $-1,25$ & 0,37 & $-2,51$ & $-1,30$ & $-1,33$ & $-2,22$ \\
\hline$\sigma_{3}$ & $-0,41$ & $-0,76$ & $-0,66$ & $-1,43$ & $-0,33$ & $-0,72$ & $-0,53$ & $-0,90$ & $-1,02$ \\
\hline$\sigma_{4}$ & 0,49 & $1,97 *$ & 0,47 & 0,26 & 0,45 & $4,13 *$ & 0,78 & 0,69 & 0,92 \\
\hline $\bar{\sigma}_{5}$ & 1,25 & 1,12 & 0,08 & 0,43 & 1,57 & 0,25 & 0,73 & 1,45 & 1,37 \\
\hline$\sigma_{6}$ & 1,20 & 1,15 & 1,20 & 0,66 & 1,20 & 1,85 & 0,39 & 1,03 & 0,88 \\
\hline$\sigma_{7}$ & $-0,40$ & $-2,02$ & $-0,92$ & $-1,79$ & $-0,48$ & $-2,39$ & $-0,11$ & $-1,00$ & $-2,14$ \\
\hline $\bar{\sigma}_{8}$ & 0,37 & $1,91 *$ & 0,41 & $-0,51$ & 0,59 & $3,73 *$ & 0,01 & 0,61 & 0,50 \\
\hline$\sigma_{9}$ & $-1,44$ & $-1,72$ & $-2,25^{*}$ & $-1,39$ & $-1,11$ & $-1,74$ & $-1,18$ & $-1,67$ & $-3,08$ \\
\hline$\sigma_{10}$ & $-0,10$ & 0,16 & $-0,21$ & $-0,14$ & $-0,53$ & $-1,61$ & $-1,20$ & 0,57 & $-0,01$ \\
\hline$\sigma_{11}$ & $-0,78$ & $-2,55$ & $-0,68$ & $-0,13$ & $-0,70$ & $-3,69 *$ & $-0,10$ & 0,14 & $-2,02$ \\
\hline$\sigma_{12}$ & $-0,73$ & $-0,99$ & $-0,14$ & 0,03 & 0,09 & 0,58 & $-0,47$ & $-0,34$ & 0,22 \\
\hline \multicolumn{10}{|l|}{ Estadíst-F } \\
\hline$\sigma_{3}=\sigma_{4}$ & 0,21 & 2,32 & 0,85 & 1,06 & 0,16 & $9,58 *$ & 0,50 & 0,69 & 1,14 \\
\hline$\sigma_{5}=\sigma_{6}$ & 1,72 & 1,48 & 1,48 & 0,34 & 2,27 & 1,79 & 0,38 & 1,86 & 1,45 \\
\hline$\sigma_{7}=\sigma_{8}$ & 0,13 & 4,64 & 4,64 & 1,73 & 0,28 & $11,75^{*}$ & 0,01 & 0,65 & 2,89 \\
\hline$\sigma_{9}=\sigma_{10}$ & 1,04 & 1,51 & 1,51 & 0,97 & 0,70 & 2,82 & 1,27 & 1,60 & 4,75 \\
\hline$\sigma_{11}=\sigma_{12}$ & 0,54 & 3,57 & 3,57 & 0,01 & 0,25 & $7,32 *$ & 0,11 & 0,07 & 2,08 \\
\hline$\sigma_{3}=\sigma_{4}=\sigma_{12}$ & 0,79 & 2,93 & 2,93 & 0,85 & 0,83 & $7,81 *$ & 0,34 & 1,16 & 2,86 \\
\hline \multicolumn{10}{|c|}{ SERVICIOS DE URGENCIA HOSPITALARIOS (SUH) } \\
\hline \multicolumn{10}{|l|}{ Estadíst-t: } \\
\hline$\sigma_{1}$ & 1,12 & 0,87 & 1,38 & 1,22 & 1,04 & 0,22 & 1,22 & $2,48^{*}$ & 0,85 \\
\hline$\sigma_{2}$ & $-0,12$ & $-0,77$ & $-2,85^{*}$ & $-1,91$ & $-0,92$ & $-0,81$ & $-1,01$ & $-0,89$ & $-0,84$ \\
\hline$\sigma_{3}$ & $-1,10$ & 0,27 & $-1,81$ & $-0,89$ & $-0,87$ & $-1,48$ & $-1,03$ & $-0,82$ & $-1,07$ \\
\hline$\sigma_{4}$ & 0,17 & 1,10 & $2,03^{*}$ & 0,79 & 0,57 & 1,15 & 0,78 & 1,56 & 0,59 \\
\hline$\sigma_{5}$ & $-0,49$ & 0,62 & $-0,02$ & $-0,22$ & 0,67 & 0,35 & 1,14 & 0,85 & 0,55 \\
\hline$\sigma_{6}$ & $-0,17$ & 1,09 & $2,77^{*}$ & $2,64 *$ & 0,34 & $-0,45$ & $-0,55$ & 0,45 & $1,64 *$ \\
\hline$\sigma_{7}$ & $-0,98$ & $-1,39$ & $-2,07$ & $-1,52$ & $1,94 *$ & 0,77 & $-0,95$ & $-0,50$ & $-1,01$ \\
\hline$\sigma_{8}$ & 1,59 & 0,30 & 1,00 & 1,13 & $-0,75$ & $-0,59$ & 1,41 & 0,91 & 0,60 \\
\hline$\sigma_{9}$ & 0,71 & $-1,01$ & $-3,20$ & $-1,55$ & 0,34 & 0,27 & $-1,44$ & $-0,60$ & $-1,72$ \\
\hline$\sigma_{10}$ & 0,15 & $-0,28$ & 0,67 & 1,40 & $-1,42$ & $-1,08$ & $-0,62$ & $-0,44$ & $-0,82$ \\
\hline$\sigma_{11}$ & $-0,42$ & $-1,17$ & $-1,70$ & $-1,73$ & $-1,39$ & $-0,28$ & $-0,15$ & $-0,49$ & $-0,53$ \\
\hline$\sigma_{12}$ & $-0,04$ & $-0,23$ & $-0,28$ & $-1,45$ & $-1,13$ & 0,46 & 0,66 & $-1,35$ & $-0,55$ \\
\hline \multicolumn{10}{|l|}{ Estadíst-F } \\
\hline$\sigma_{3}=\sigma_{4}$ & 0,64 & 0,65 & 3,70 & 0,77 & 0,55 & 1,84 & 0,90 & 1,60 & 0,75 \\
\hline$\sigma_{5}=\sigma_{6}$ & 0,15 & 0,88 & 3,84 & 3,50 & 2,28 & 0,17 & 0,73 & 0,50 & 1,58 \\
\hline$\sigma_{7}=\sigma_{8}$ & 1,76 & 0,97 & 2,79 & 1,90 & 0,31 & 0,42 & 1,29 & 0,49 & 0,64 \\
\hline$\sigma_{9}=\sigma_{10}$ & 0,25 & 0,50 & 5,54 & 2,45 & 1,21 & 0,64 & 1,09 & 0,26 & 1,53 \\
\hline$\sigma_{11}=\sigma_{12}$ & 0,09 & 0,70 & 1,47 & 2,37 & 1,49 & 0,16 & 0,25 & 0,99 & 0,25 \\
\hline$\sigma_{3}=\sigma_{4}=\sigma_{12}$ & 0,78 & 1,22 & 3,38 & 3,25 & 1,30 & 0,59 & 0,87 & 0,80 & 1,07 \\
\hline
\end{tabular}

Notas: n: regresión sin constante ni tendencia; c,d : regresión con constante y dummies estacionales; c,t,d: regresión con constante, tendencia y dummies estacionales * Significativo al 5\% (se rechaza la hipótesis nula). 
Tabla 4

Resultados del contraste de cointegración de Johansen

\begin{tabular}{|c|c|c|c|c|c|c|}
\hline Área Sanitaria & $\mathbf{H}_{0}$ & $\mathbf{H}_{\mathbf{a}}$ & $\operatorname{VAR}(\mathbf{p})$ & $\begin{array}{c}\text { Test de la } \\
\operatorname{traza}-2 \operatorname{Ln}\left(Q^{*}\right)\end{array}$ & $\begin{array}{c}\text { Test de la } \\
\operatorname{traza}-2 \operatorname{Ln}(Q)\end{array}$ & $\delta$ \\
\hline I Jarrio & $\begin{array}{l}r=0 \\
r=1\end{array}$ & $\begin{array}{l}r=1 \\
r=2\end{array}$ & $\begin{array}{l}6 \\
6\end{array}$ & $\begin{array}{c}2.223 * \\
597\end{array}$ & $\begin{array}{r}2.140^{*} \\
556^{*}\end{array}$ & $\begin{array}{l}078 \\
(244)^{\mathrm{TM}}\end{array}$ \\
\hline II Cangas del Narcea & $\begin{array}{l}r=0 \\
r=1\end{array}$ & $\begin{array}{l}r=1 \\
r=2\end{array}$ & $\begin{array}{l}4 \\
4\end{array}$ & $\begin{array}{r}1.356 \\
325\end{array}$ & $\begin{array}{r}1.338 \\
325\end{array}$ & - \\
\hline III Avilés & $\begin{array}{l}r=0 \\
r=1\end{array}$ & $\begin{array}{l}r=1 \\
r=2\end{array}$ & $\begin{array}{l}4 \\
4 \\
\end{array}$ & $\begin{array}{c}2.192 * \\
542\end{array}$ & $\begin{array}{r}2.184^{*} \\
539^{*} \\
\end{array}$ & $\begin{array}{l}0066 \\
(177)^{\mathrm{TM}} \\
\end{array}$ \\
\hline IV Oviedo & $\begin{array}{l}r=0 \\
r=1\end{array}$ & $\begin{array}{l}r=1 \\
r=2\end{array}$ & $\begin{array}{l}4 \\
4\end{array}$ & $\begin{array}{c}2.391 * \\
345\end{array}$ & $\begin{array}{c}2.381^{*} \\
334\end{array}$ & $\begin{array}{l}-0271 \\
(220)^{\mathrm{TM}}\end{array}$ \\
\hline V Gijón & $\begin{array}{l}\mathrm{r}=0 \\
\mathrm{r}=1\end{array}$ & $\begin{array}{l}r=1 \\
r=2\end{array}$ & $\begin{array}{l}6 \\
6\end{array}$ & $\begin{array}{c}2.030 * \\
832\end{array}$ & $\begin{array}{r}2.025^{*} \\
831^{*}\end{array}$ & $\begin{array}{l}0045 \\
(162)^{\mathrm{TM}}\end{array}$ \\
\hline VI Arriondas & $\begin{array}{l}r=0 \\
r=1\end{array}$ & $\begin{array}{l}r=1 \\
r=2\end{array}$ & $\begin{array}{l}4 \\
4\end{array}$ & $\begin{array}{l}913 \\
153\end{array}$ & $\begin{array}{l}792 \\
054\end{array}$ & - \\
\hline VII Nalón & $\begin{array}{l}r=0 \\
r=1\end{array}$ & $\begin{array}{l}r=1 \\
r=2\end{array}$ & $\begin{array}{l}5 \\
5\end{array}$ & $\begin{array}{r}1.878 \\
643\end{array}$ & $\begin{array}{c}1.868^{*} \\
640\end{array}$ & - \\
\hline Total Asturias & $\begin{array}{l}r=0 \\
r=1\end{array}$ & $\begin{array}{l}r=1 \\
r=2\end{array}$ & $\begin{array}{l}6 \\
6\end{array}$ & $\begin{array}{r}1.522 \\
701\end{array}$ & $\begin{array}{r}1.516 \\
701\end{array}$ & - \\
\hline
\end{tabular}

NOTAS: VAR(p) se refiere al orden elegido del esquema vectorial autorregresivo.

* Significativo al $5 \%$ (rechazo de la $\mathrm{H}_{0}$ ).

${ }^{\mathrm{TM}}$ Estadístico-t entre paréntesis.

Tabla 5

Resultados de cointegración en la relación estática Contraste de Engle y Granger (ADF)

\begin{tabular}{|c|c|c|c|}
\hline Regresión: & \multicolumn{3}{|c|}{$\log \mathbf{S U H}=\sigma+\delta \log P A C+m_{t}$} \\
\hline Área Sanitaria & б & \& & ADF \\
\hline I Jarrio & 004 & $015(452)^{\mathrm{TM}}$ & -355 \\
\hline II Cangas del Narcea & 003 & $0024(051)^{\mathrm{TM}}$ & -278 \\
\hline III Avilés & 006 & $0099(406)^{\mathrm{TM}}$ & $-435^{*}$ \\
\hline IV Oviedo & -0005 & $0143(340)^{\mathrm{TM}}$ & $-302 *$ \\
\hline V Gijón & 0035 & $0117(325)^{\mathrm{TM}}$ & $-392 *$ \\
\hline VI Arriondas & 0113 & $-0008(-011)^{\mathrm{TM}}$ & -257 \\
\hline VII Nalón & 0014 & $0048(174)^{\mathrm{TM}}$ & -235 \\
\hline Total Asturias & 0021 & $028(710)^{\mathrm{TM}}$ & -214 \\
\hline
\end{tabular}

NOTAS: * Significativo al $5 \%$ (rechazo de la $\mathrm{H}_{0}=$ no cointegración).

${ }^{\mathrm{TM}}$ Estadístico-t entre paréntesis.

tasas brutas de visitas en todas las áreas sanitarias.

En la figura 1 se observa que el crecimiento en AP fue más acusado hasta 1997, coincidiendo con un crecimiento más lento en los SUH. A partir de 1997 las urgencias crecieron de forma paralela en ambos niveles asistenciales. En el Figura 2 se observa una clara estacionalidad de las visitas urgentes sobre todo en los PAC.
En el análisis de las series temporales de visitas urgentes a los SUH y a los PAC para las ocho Áreas Sanitarias, y para el conjunto de Asturias, el carácter no estacionario de las series y su posible estacionalidad puede verse en los Figuras 2 y 3 y los resultados de los contrates estadísticos que lo corroboran en la Tabla 2.

En Asturias y las AS de Cangas del Narcea, Arriondas, Mieres y Nalón no se detecta relación de cointegración alguna; en Jarrio, Avilés y Gijón existe una relación estable; en el AS de Mieres no hay relación de cointegración puesto que presentan un orden de integración en la frecuencia cero diferente. Por ello, las visitas de los PAC son independientes de las SUH o existe una relación positiva que indica complementariedad, por lo que no hay evidencia empírica que sustente la idea de que los servicios de urgencia en atención primaria contribuyan a reducir las visitas urgentes en los hospitales; es decir, no existe capacidad de sustitución entre los dos niveles asistenciales (tablas 3 y 4). En el AS de Oviedo existe una relación de cointegración con signo negati- 
Figura 2

Evolución mensual de las visitas urgentes en Asturias y en las Áreas sanitarias I-VIII (1994-2001)
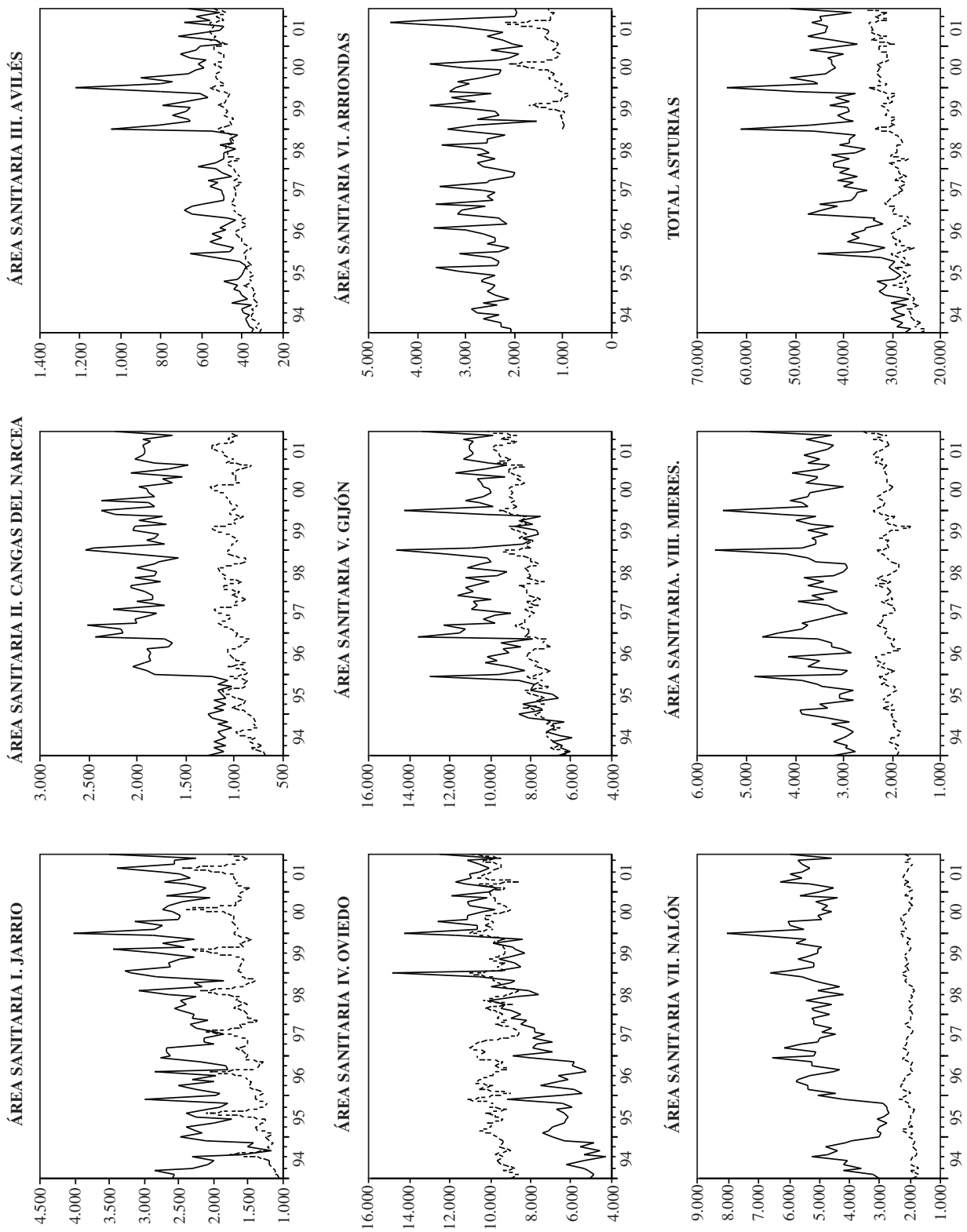
vo $(b=-2,71)$, por lo que un crecimiento del $10 \%$ en las visitas urgentes a los PAC reduciría en un $2,7 \%$ las visitas en el SUH (tabla 3).

\section{DISCUSIÓN}

En el análisis de las series temporales de las visitas urgentes para el conjunto de Asturias y para las áreas sanitarias -excepto la de Oviedo- no se ha detectado ninguna relación de cointegración, por lo que no hay evidencia empírica que sustente la idea de que los servicios de urgencia en atención primaria contribuyan a reducir las visitas urgentes en los hospitales. Es decir, no existe capacidad de sustitución entre los dos niveles asistenciales, ceteris paribus el resto de variables que influyen en la conducta de los usuarios. Estos resultados son consistentes con los obtenidos en otros estudios que analizan la efectividad de la atención continuada, de los walk-in centres y de los out-of hours GP clinics ${ }^{12,14-16 .}$

En el área sanitaria de Oviedo se ha observado un cierto grado de sustituibilidad entre los servicios de urgencias hospitalarias y de atención primaria (un crecimiento del $10 \%$ en las visitas urgentes a los PAC reduciría en un $2,7 \%$ las visitas en el SUH). Este resultado si bien necesitaría un estudio más exhaustivo, podría deberse a las características peculiares de este área, como son a nivel hospitalario: a) es el hospital de referencia para Asturias (nivel 4), b) cuenta con tres servicios de urgencias diferenciados físicamente (general, materno-infantil y silicosis), c) atiende el mayor número bruto de visitas y tiene la tasa de visitas/1000 habitantes más elevada, d) parte del mayor número de visitas al principio del periodo de estudio; e) el incremento de la tasa de visitas es el más bajo, f) hasta el año 1988 era el SUH de referencia del área sanitaria de Arriondas (en este año se inauguró el hospital comarcal de referencia para este área); y en atención pri- maria: a) es el área con mayor población, b) cuenta con mayor número de PAC y c) el incremento de la tasa de visitas en los PAC es el más alto.

La tasa de visitas urgentes ha sufrido un fuerte incremento en Asturias, más acusado en atención primaria que en los hospitales y este crecimiento no es homogéneo en todas las áreas sanitarias. Esto, unido a las diferencias de la tasa de visitas observadas entre áreas sanitarias, implica que el riesgo de utilizar los servicios de urgencia, sean de atención primaria u hospitalarios, es distinto, sin que se conozcan los posibles beneficios/perjuicios de una mayor o menor utilización de los servicios urgentes.

Aunque la variabilidad sea menor que en otras modalidades de atención sanitaria $^{25-27}$, el volumen de visitas urgentes convierte estas variaciones en un problema de gran magnitud, si se aplicase la tasa de visitas hospitalarias del Área del Nalón en el año 2001 a la de Oviedo supondría una disminución de 28.316 visitas $(23,7 \%)$, si se hace a la inversa el incremento de visitas en el Nalón sería de 5.818 (21,5\%). Si se hace el mismo cálculo en atención primaria el incremento en Oviedo seria de 123.051 visitas $(107,1 \%)$ y la disminución en el Nalón de 11.858 (27,6\%).

Entre las limitaciones de este trabajo hay que señalar que el análisis realizado asume que los factores que influyen sobre el comportamiento de los usuarios respecto a la utilización de los servicios de urgencia no ha variado de forma sustancial, esto es, que no existen otros factores que hayan estimulado la utilización de las urgencias hospitalarias, compensando la posible reducción causada por la apertura de los PAC. Aunque se trata de una limitación siempre presente en los estudios observacionales, la estabilidad del incremento en las tasas de utilización de los servicios hospitalarios -con independencia de las 
tasas de atención primaria- no avala la hipótesis de la presencia de otras intervenciones que hayan modificado el sentido de las tendencias. De otro lado, mientras que el denominador utilizado para calcular las tasas es censal, no todos los pacientes acuden a los SUH de sus áreas de residencia, aspecto que puede suponer una fuente de variación sobreañadida. Igualmente, sólo se incluyen las visitas a centros de la red pública, aunque dada la estructura sanitaria de Asturias (sólo un hospital privado dispone de servicio de urgencias) la repercusión de este aspecto se prevé mínima.

En todo caso, este estudio sugiere que el aumento de la oferta de servicios de urgencia en atención primaria incrementa la utilización de estos servicios, sin disminuir la utilización de los SUH, levantado serias dudas sobre la capacidad de las estrategias de mejorar la accesibilidad a los centros de atención primaria para reducir -a medio/largo plazo- las visitas a los SUH, independientemente de que la mayor accesibilidad a las urgencias en atención primaria, en si misma, mejore la calidad de la asistencia y la satisfacción del la población. Dado el escaso éxito de las intervenciones realizadas para disminuir el uso inadecuado de los SUH cobran fuerza las propuestas que optan por redefinir los propios SUH. Para varios autores $^{9,}$ 28-31 estos servicios, tal y como están concebidos hoy en día, no son adecuados para el tipo de asistencia que solicita la población y las políticas deberían dirigirse hacia su rediseño para que estén en condiciones de atender los casos urgentes y no urgentes en un tiempo razonable y con costes también razonables.

\section{AGRADECIMIENTOS}

A las gerencias de las Áreas de Atención Primaria de Asturias y a los hospitales Álvarez Buylla de Mieres, Jove de Gijón y Grande Covián de Arriondas, por facilitar los datos sobre las visitas urgentes atendi- das y su colaboración para responder y aclarar cuantas dudas se les han planteado.

\section{BIBLIOGRAFÍA}

1. Alberti G. Transforming Emergency Care in England. London: Department of Health; 2004. Disponible en: http://www.dh.gov.uk/PublicationsAndStatistics/Publications/PublicationsPolicyA ndGuidance/PublicationsPolicyAndGuidanceArticle/fs/en?CONTENT_ID=4091775\&chk=9mg n5R. [Citado el 1/3/2007].

2. Cooke M, Fisher J, Dale J, McLeod E, Szczepura A, Walley P, et al. Reducing Attendances and Waits in A\&E Departments:A review and Survey of Present Innovations'. London: NHS, Service Development Organisation; 2004.

3. Bond K, Ospina M, Blitz S, Friesen C, Innes G, Yoon $\mathrm{P}$, et al. Interventions to reduce overcrowding in emergency departments [Technology report no 67.4]. Ottawa: Canadian Agency for Drugs and Technologies in Health; 2006.

4. Health Services Utilization and Research Committee. Reducing non-urgent use of the emergency department: a review of strategies and guide for future research. Saskatoon: Health Services Utilization and Research Committee; 1997.

5. New Zealand Health Technology Assessment. Emergency Department Attendance. A critical appraisal of the key literature. Christchurch: New Zealand Health Technology Assessment Clearing House; 1998.

6. Peiró, S, Sempere, T y Oterino de la Fuente, D. Efectividad de las intervenciones para reducir la utilización inapropiada de los servicios hospitalarios de urgencias. Revisando la literatura 10 años después del Informe del Defensor del Pueblo. Economía y Salud 1999; 33: 1-16.

7. Alberola, V, y Rivera, F. La Atención Primaria como determinante de la utilización del Servicio de Urgencias Hospitalario. Aten Primaria 1994; 6: 825-828.

8. Gill, J M y Diamond, JJ. Effect of Primary Care Referral on Emergency Department Use: Evaluation of a Statewide Medicaid Program. Del Med J 1996; 68: 437-442.

9. Lowe, RA, Russell Localio, Schwarz, DF, Williams, S, Wolf Tuton, L Maroney, S et al. Association Between Primary Care Practice Characte- 
ristics and Emergency Department Use in a Medicaid Managed Care Organization. Med Care 2005; 43: 792-800.

10. Bolivar, I, Balanzo, X, Armada, A, Fernández, J L, Foz, G, Sanz E et al. El impacto de la reforma de la Atención Primaria en la utilización de Servicios de Urgencias Hospitalarios. Med Clin 1996; 107: 289-295.

11. Chapman, JL, Zechel, A, Carter, YH y Abbott, S. Systematic review of recent innovations in service provision to improve access to primary care. Br J General Practice 2004; 54: 374-381.

12. Oterino de la Fuente, D, Baños Pino, J, Fernández Blanco, V y Rodríguez-Álvarez, A. Does Better Access to Primary Care Reduce Utilisation of Hospital Accident and Emergency Departments? A Time-Series Analysis, Eur J Public Health 2006; Jun 3 [Epub ahead of print].

13. Valdrés P, Acitores JM, González A, Rubio, L. I. Impacto sobre la asistencia en las urgencias hospitalarias de la implantación de la atención continuada en los centros de salud de Logroño. Aten Primaria 1993; 4: 178-180.

14. Chalder M, Sharp D, Moore L, Salisbury C. Impact of NHS walk-in centres on the workload of other local healthcare providers: time series analysis. Br Med J 2003; 326: 532-536.

15. Jones M. Walk-in Primary Medical Care Centres: Lessons from Canada. Br Med J 2000; 321: 928-931.

16. Salisbury C y Munro, J. Walk-in centres in primary care: a review of the international literature. Br J General Practice 2003; 53: 53-59.

17. McCusker J, Verdon J. Do Geriatric Interventions Reduce Emergency Department Visits? A Systematic Review. J Gerontol a Biol Sci Med Sci 2006; 61: 53-62.

18. Ministerio de Sanidad y Consumo. Asistencia especializada Actividad 2001 Evolución de indicadores 1991-2001. Instituto Nacional de la Salud Subdirección General de Coordinación Administrativa; 2002.

19. Matea ML. Contrastes de raíces unitarias para series mensuales Una aplicación al IPC. Revista Española de Economía 1994; 11: 7-25.

20. Hylleberg S, Engle RF, Granger CW, Yoo BS. Seasonal Integration and Co-Integration. J Econom 1990; 44: 215-228.
21. Engle RF and Granger CWJ. Co-integration and error correction representation, estimation, and testing. Econometrica 1987; 55: 251- 76.

22. Johansen S. Statistical Analysis of Cointegration Vectors. J Econ Dyn Control 1988; 12: 231-254.

23. Reimers HE. Comparisons of Tests for Multivariate Cointegration. Statistical Papers 1992; 33: 335-359.

24. Banerjee A, Dolado J, Galbraith J, Hendry DF. Co-Integration, Error-Correction and the Econometric Analysis of Non-Stationary Data. Oxford University Press; 1993.

25. Librero J, Peiró S, Bernal-Delgado E, Rivas F, Martínez N, Sotoca R, Ridao M, Castaño E Grupo VPM-SNS. Variaciones en Intervenciones de Cirugía General en el Sistema Nacional de Salud. Atlas Var Pract Med Sist Nac Salud 2005; 2: 63-81.

26. Marqués JA, Peiró S, Medrano J, Librero J, Meneu R y López Reneo R. Variabilidad en la práctica clínica Disponibilidad de recursos y utilización de procedimientos quirúrgicos. Gestión Hospitalaria 2001; 12: 196-201.

27. Oterino de la Fuente D, Castaño E, Librero J, Peiró S, Bernal-Delgado E, Martínez N, Rivas F, Atienza Merino G. Grupo VPM-SNS. Variaciones en hospitalizaciones pediátricas por procedimientos quirúrgicos y diagnósticos seleccionados. Atlas Var Pract Med Sist Nac Salud 2006; 3: 101-116.

28. Murphy AW Inappropriate attenders at accident and emergency departments Definition, incidence and reasons for attendance. Fam Pract 1998; 15: 23-35.

29. Miro O, Sánchez M, Mestre G, Coll-Vinent B, Bragulat E, Espinosa G, Soler N, Gotsens R, Milla J. Evaluación del impacto en la calidad asistencial y análisis coste-efectividad de la reforma de un servicio de urgencias de medicina. Med Clin 2001; 117: 7-11.

30. Dale J, Green J, Reid F y Glucksman E. Primary care in the accident and emergency department: I Prospective identification of patients. Br Med J 1995; 311: 423-426.

31. Aranaz JM, Martínez R, Rodrigo V, Gómez F, Antón P. Adecuación de la demanda de atención sanitaria en servicios de urgencias hospitalarios. Med Clin 2004; 123: 615-618. 